\title{
APPROXIMATIONS TO THE NORM OF THE SINGULAR PART OF A MEASURE
}

\author{
by LOUIS PIGNO
}

(Received 6th August 1974)

Let $G$ be a non-discrete LCA group with dual group $\Gamma$. Denote by $M(G)$ the usual convolution algebra of bounded Borel measures on $G$ and $M_{a}(G)$ those $\mu \in M(G)$ which are absolutely continuous with respect to $m_{G}$-the Haar measure on $G$.

The Fourier-Stieltjes transform $\hat{\mu}$ of a measure $\mu \in M(G)$ is defined by

$$
\hat{\mu}(\gamma)=\int_{G}(-x, \gamma) d \mu(x) \quad(\gamma \in \Gamma) .
$$

A closed subset $\mathscr{R}$ of $\Gamma$ is called a Riesz (or small) set if whenever supp $\hat{\mu} \subset \mathscr{R}$ then $\mu \in M_{a}(G)$. In this paper we pursue a line of research originated by R. Doss in (3) and subsequently explicated by him in (5).

Suppose $p(x)=\sum_{n} a_{n}\left(-x, \gamma_{n}\right)$ is a trigonometric polynomial on $G$ with $\|p\|_{\infty} \leqq 1$. We shall concern ourselves with calculating the "limit" of $\sum_{n} a_{n} \hat{A}\left(\gamma_{n}\right)$ off the Riesz set $\mathscr{R}$. We need to make our idea for taking such a limit precise:

Forany subset $\mathscr{S} \subset \Gamma$ denote by $P_{\mathscr{S}}$ the set of all trigonometric polynomials on\} G \text { of the form } p ( x ) = \sum _ { n } a _ { n } ( - x , \gamma _ { n } ) \text { with } \| p \| _ { \infty } \leqq 1 \text { and } \gamma _ { n } \notin \mathscr { S } \text { . Define }

$$
\|\mu\|_{\mathscr{S}}=\sup _{p \in P_{\mathscr{S}}}\left|\sum_{n} a_{n} \hat{\mu}\left(\gamma_{n}\right)\right| \text {. }
$$

Then by the limit of $\sum_{n} a_{n} \hat{\alpha}\left(\gamma_{n}\right)$ off $\mathscr{S}$ we mean

$$
\lim _{\boldsymbol{K} \rightarrow \infty}\|\mu\|_{\mathcal{S O}_{\cup K}}=\inf _{\boldsymbol{K}}\|\mu\|_{\mathscr{S} \cup \mathbf{K}} \quad(\boldsymbol{K} \text { compact in } \Gamma) .
$$

For $\mathscr{R}$, a Riesz set, put

$$
\lim _{\mathscr{R} \rightarrow \infty}\|\mu\|_{\mathscr{R}}=\inf _{\mathscr{R}}\|\mu\|_{\mathscr{R}} \text {. }
$$

Note that in this general setting the methods of (5) are not available. Our main result now follows; the proof is pleasantly simple.

Theorem 1. Let $\mu \in M(G)$ and let $\mu=\mu_{a}+\mu_{s}$ be the canonical Lebesgue decomposition of $\mu$ with $\mu_{a} \in M_{a}(G)$ and $\mu_{s}$ singular with respect to $m_{G}$. Then

$$
\lim _{\mathfrak{R} \rightarrow \infty}\|\mu\|_{\mathbb{R}}=\lim _{\mathbf{X} \rightarrow \infty}\|\mu\|_{\mathfrak{Q} \cup \mathrm{K}}=\left\|\mu_{s}\right\| \text {. }
$$


Proof. Let $\mathscr{R}$ be any Riesz set. Then

$$
\|\mu\|_{\mathscr{R}} \leqq\left\|\mu_{s}\right\|+\left\|\mu_{a}\right\|_{\mathscr{R}}
$$

and since $\lim _{\mathscr{R} \rightarrow \infty}\left\|\mu_{a}\right\|_{\mathscr{R}}=0$ (this is easy to check), we gather

$$
\lim _{\mathscr{R} \rightarrow \infty}\|\mu\|_{\mathscr{R}} \leqq\left\|\mu_{s}\right\| .
$$

To prove the opposite inequality we obtain via Proposition 2 and the Main Theorem of (4) a measure $\lambda_{\mathscr{R}} \in M(G)$ such that

and

$$
\hat{\lambda}_{\mathscr{R}}=\hat{\mu} \quad \text { off } \mathscr{R}
$$

Observe that the identity

$$
\left\|\lambda_{\mathscr{R}}\right\| \leqq\|\mu\|_{\mathscr{R}} \text {. }
$$

$$
\mu_{s}+\mu_{a}-\left(\mu-\lambda_{\mathscr{R}}\right)=\lambda_{\mathscr{R}}
$$

when combined with (2) yields the inequality

$$
\left\|\mu_{s}\right\| \leqq\left\|\lambda_{\mathscr{R}}\right\| \text {. }
$$

By (3) we must have $\left\|\mu_{s}\right\| \leqq\|\mu\|_{\mathscr{R}}$. Thus

$$
\left\|\mu_{s}\right\| \leqq\|\mu\|_{\mathscr{R}} \text { for all Riesz sets } \mathscr{R} \text {. }
$$

Hence (1) and (4) give

$$
\lim _{\mathscr{R} \rightarrow \infty}\|\mu\|_{\mathscr{R}}=\left\|\mu_{s}\right\|
$$

Finally, let $K$ be any compact subset of $\Gamma$. We claim that $\mathscr{R} \cup K$ is a Riesz set. For suppose supp $\hat{v} \subset \mathscr{R} \cup K, v \in M(G)$. Then there is an $f \in L^{1}(G)$ such that $\hat{f}(K)=1$. Since $v-f * v \in M_{a}(G)$, this implies $v \in M_{a}(G)$. We conclude therefore, by (4), that

$$
\left\|\mu_{s}\right\| \leqq\|\mu\|_{\text {RuK }} \text {. }
$$

Since $\lim _{K \rightarrow \infty}\left\|\mu_{a}\right\|_{\mathscr{R} \cup K}=0$, it follows that $\lim _{K \rightarrow \infty}\|\mu\|_{\mathscr{R} \cup K}=\left\|\mu_{s}\right\|$ and the theorem is proved.

Corollary 1. A measure $\mu \in M(G)$ is absolutely continuous if and only if

$$
\lim _{\mathbf{K} \rightarrow \infty}\|\mu\|_{\operatorname{BQ} \cup \mathbf{K}}=0 \text {. }
$$

Corollary 2. A measure $\mu \in M(G)$ is singular if and only if

$$
\lim _{\mathbf{K} \rightarrow \infty}\|\mu\|_{\mathscr{R} \cup \mathbf{K}}=\|\mu\| \text {. }
$$

Next we give an extension of a Theorem of I. Glicksberg (6, p. 419):

Theorem 2. Let $\mathscr{S}$ be any closed subset of $\Gamma$ such that $\mathscr{S} \cap(\gamma-\mathscr{S})$ has finite Haar measure for a dense subset of $y \in \Gamma$ and let $\mu_{i} \in M(G) i \in\{1,2\}$. Suppose

$$
\lim _{\mathbf{K} \rightarrow \infty}\left\|\mu_{i}\right\|_{\mathscr{S} \cup \mathbf{K}}=0 \text {. }
$$


Then $\mu_{1} * \mu_{2}$ is absolutely continuous with respect to $m_{G}$.

Proof. Given $\varepsilon>0$, there correspond compact $K_{i \varepsilon}$ such that for all trigonometric polynomials of the form $p(x)=\sum_{n} c_{n}\left(-x, \gamma_{n}\right)$ with $\|p\|_{\infty} \leqq 1$ and $\gamma_{n} \notin \mathscr{S} \cup K_{i \varepsilon}$ the inequality

obtains.

$$
\left|\Sigma c_{n} \hat{\mu}_{i}\left(\gamma_{n}\right)\right|<\varepsilon
$$

Now (5), Proposition 2 and the Main Theorem of (4) give a measure $\mu_{i \varepsilon} \in M(G)$ such that

and

$$
\hat{\mu}_{i}=\hat{\mu}_{i \varepsilon} \quad \text { off } \mathscr{S} \cup K_{i \varepsilon}
$$

$$
\text { i } \mu_{i \varepsilon} \| \leqq \varepsilon .
$$

Recall that there exist $f_{i} \in L^{1}(G)$ such that $\hat{f}_{i}\left(K_{i \varepsilon}\right)=1$. Observe that

$$
\operatorname{supp}\left\{\left(\mu_{i}-\mu_{i \varepsilon}\right)-f_{i} *\left(\mu_{i}-\mu_{i e}\right)\right\}^{\wedge} \subset \mathscr{S}
$$

which implies by Glicksberg's Theorem that

$$
\left\{\left(\mu_{1}-\mu_{1 \varepsilon}\right)-f_{1} *\left(\mu_{1}-\mu_{1 \varepsilon}\right)\right\} *\left\{\left(\mu_{2}-\mu_{2 \varepsilon}\right)-f_{2} *\left(\mu_{2}-\mu_{2 \varepsilon}\right)\right\} \in M_{a}(G) .
$$

Thus

$$
\left(\mu_{1}-\mu_{1 \varepsilon}\right) *\left(\mu_{2}-\mu_{2 \varepsilon}\right) \in M_{a}(G) .
$$

Let $\varepsilon \rightarrow 0$ in (8). From (7) we infer that $\mu_{1} * \mu_{2} \in M_{a}(G)$. This completes the proof.

Remarks. (1) Theorem 1 and proof (with obvious modification) holds true for compact groups (i.e. not necessarily abelian).

(2) To obtain Doss' results in (3) and (5), it suffices to take $\mathscr{R}$ compact in Theorem 1.

(3) For some interesting examples of Riesz sets when $G=T$ the reader is referred to (1) and (7).

(4) Using the same technique as in Theorem 2, one can give the following extension of a Theorem of Doss (2, p. 81):

Suppose $G$ is a locally compact abelian group whose dual $\Gamma$ is ordered, $\mu \in M(G)$ and $\lim _{\boldsymbol{K} \rightarrow \infty}\|\mu\|_{\mathscr{P} \cup K}=0$ where $\mathscr{P}$ is the positive cone of $\Gamma$. Then $\hat{\mu}_{s}$ is of analytic type and $\hat{\mu}_{s}(0)=0$.

(5) Let $-N$ be the negative integers and $S$ any set of integers $\left\{n_{k}\right\}$ where $\lim _{k \rightarrow \infty}\left(n_{k+p}-n_{k}\right)=0$. Suppose

$$
\lim _{\boldsymbol{K} \rightarrow \infty}\|\mu\|_{-\boldsymbol{N} \cup S \cup \boldsymbol{K}}=0 .
$$

Then it can be shown that the $p+1$ convolution power $\mu^{p+1} \in M_{a}(T)$ (see (8)).

The author takes pleasure in thanking Sadahiro Saeki for helpful conversations. 


\section{REFERENCES}

(1) R. Dressler and L. Pigno, On strong Riesz sets, Colloq. Math. 29 (1974), 157-158.

(2) R. Doss, On the Fourier-Stieltjes transforms of singular or absolutely continuous measures, Math. Z. 97 (1967), 77-84.

(3) R. Doss, On the transform of a singular or absolutely continuous measure, Proc. Amer. Math. Soc. 19 (1968), 361-363.

(4) R. Doss, Approximations and representations for Fourier transforms, Trans. Amer. Math. Soc. 153 (1971), 211-221.

(5) R. Doss, Harmonic analysis of oscillations, J. London Math. Soc. 7 (1973), 41-47.

(6) I. GLiCKsberg, Fourier-Stieltjes transforms with small supports, Illinois J. Math. 9 (1965), 418-427.

(7) Y. MEYER, Spectres des mesures et mesures absolument continues, Studia Math. 30 (1968), 87-99.

(8) L. J. Wallen, Fourier-Stieltjes transforms tending to zero, Proc. Amer. Math. Soc. 24 (1970), 651-652.

Kansas State University 